\title{
High pressure chemical reactivity and structural study of the Na-P and Li-P systems
}

\author{
River A. Leversee, ${ }^{a b}$ Kristen Rode, ${ }^{a c}$ Eran Greenberg, ${ }^{d}$ Vitali B. Prakapenka, ${ }^{d}$ Jesse S. \\ Smith, ${ }^{e}$ Martin Kunz,${ }^{f}$ Chris J. Pickard,${ }^{*}{ }^{g h}$ Elissaios Stavrou ${ }^{* a}$
}

Received Date

Accepted Date

DOI: $00.0000 / x x x x x x x x x x$
The Na-P and Li-P chemical systems were studied under pressure using synchrotron $x$-ray diffraction in a diamond anvil cell up to $20 \mathrm{GPa}$, combined with the AIRSS ab-initio random structure searching technique. The results reveal an enhanced reactivity of both alkali metals with phosphorous at slightly elevated pressures. This enables the synthesis of $\mathrm{Li}_{3} \mathrm{P}$ and $\mathrm{Na}_{3} \mathrm{P}$ at room temperature (RT) starting from element precursors, bypassing the established chemical synthesis methods. Both compounds undergo a pressure-induced phase transition from the hexagonal $\mathrm{Na}_{3}$ As-type structure (stable at ambient conditions) towards a $F m \overline{3} m(\mathrm{FCC})$ structure that remains stable up to $20 \mathrm{GPa}$. Attempts to synthesize compounds with higher alkali metal content (such as $\mathrm{Li}_{5} \mathrm{P}$ ) using high-temperature and -pressure conditions (up to $2000+\mathrm{K}$ and $30 \mathrm{GPa}$ ), inspired by recent theoretical predictions, were not successful.

\section{Introduction}

Novel synthesis pathways have become critically important in the development of materials that are precisely tailored to meet the need for specific properties/applications, i.e. "materials by design". One way to overcome present barriers in the synthesis of materials is to alter the fundamental chemistry that governs the relevant chemical systems. In this context, tuning the composition of a given chemical system beyond one that is thermodynamically stable at ambient conditions represents an extremely promising approach to the synthesis of novel materials. Recent experimental studies and theoretical predictions highlight that the chemical properties of elements, and thus the more stable stoichiometries of a chemical system, are not a priori the same at elevated pressures as at ambient pressure ${ }^{1,2}$. Indeed, thermodynamically stable compounds with novel compositions that challenge our traditional "textbook"picture were theoretically predicted and experimentally synthesized even in relatively simple chemical systems ${ }^{3-8}$. These

\footnotetext{
${ }^{a}$ Lawrence Livermore National Laboratory, Physical and Life Sciences Directorate, P.O. Box 808 L-350, Livermore, California 94550, USA E-mail:stavrou1@llnl.gov

${ }^{b}$ Department of Physics, Michigan Technological University, Houghton, Michigan 49931, USA

${ }^{c}$ Department of Geoscience, University of Nevada, Las Vegas, NV 89134, USA

${ }^{d}$ Center for Advanced Radiation Sources, University of Chicago, Chicago, IL 60637, USA

${ }^{e}$ High Pressure Collaborative Access Team, X-ray Science Division, Argonne National Laboratory, Argonne, Illinois 60439 USA

${ }^{f}$ Advanced Light Source, Lawrence Berkeley National Laboratory, Berkeley, CA 94720, USA

${ }^{g}$ Department of Materials Science and Metallurgy, University of Cambridge, 27 Charles Babbage Road, Cambridge CB3 OFS, United Kingdom E-mail:cjp20@cam.ac.uk

${ }^{h}$ Advanced Institute for Materials Research, Tohoku University, Sendai, Japan
}

materials can be synthesized under pressure due to the formation of chemical bonds and atomic arrangements that are absent, or even forbidden, at ambient conditions ${ }^{9}$.

In the context of novel materials, the next generation of lithium ion batteries (LIBs) and the practical realization of sodium ion batteries (NIBs) are both directly related to the challenging effort of finding suitable anode materials. These materials should simultaneously exhibit high specific capacity and small volumetric expansion to increase stability to electrochemical degradation, during lithiation and sodiation ${ }^{10-12}$. NIBs are a promising alternative to LIBs; however, the unsuitability of the traditional materials (graphite and silicon) used for LIBs hinder practical applications of NIBs. Successful synthesis of applicable anode materials can be the basis of a major breakthrough in the field.

Recent theoretical studies strongly suggest that anode materials for LIBs and NIBs based on phosphorous ${ }^{10,13-16}$ are capable of exhibiting record specific capacities (4326 mAhg-1) with much lower volumetric expansion $(<200 \%)$ than silicon via the formation of novel compounds, such as $\mathrm{Na}_{5} \mathrm{P}$ and $\mathrm{Li}_{5} \mathrm{P}$ and $\mathrm{Li}_{6} \mathrm{P}$. These compounds are predicted to be slightly above the ambient conditions convex hull (i.e. the stable compositions at a given pressure and temperature) and could be synthesized at very low pressures ${ }^{15}$. Lower synthesis pressure additionally increases the probability of metastability. For instance, compounds with $\mathrm{Na}_{5} \mathrm{P}$ and $\mathrm{Na}_{6} \mathrm{P}$ stroichiometries have formation enthalpies just above the convex hull at ambient conditions ${ }^{10}$. In addition to the possible applications of $\mathrm{Li}-\mathrm{P}$ and Na-P materials in batteries, very recently a $\mathrm{Li}_{6} \mathrm{P}$ electride was predicted that becomes a superconductor with a superconducting transition temperature Tc of $39.3 \mathrm{~K}^{17}$. 
To our knowledge, there are very limited published ${ }^{18}$ experimental results (regarding chemical reactivity or structural properties) of the Na-P and Li-P chemical systems and compounds under pressure which motivates the present study. We specifically explored: a) the possible synthesis of $\mathrm{Li}_{3} \mathrm{P}$ and $\mathrm{Na}_{3} \mathrm{P}$, using pathways that go beyond traditional chemical synthesis routes, b) the high-pressure structural behavior of $\mathrm{Li}_{3} \mathrm{P}$ and $\mathrm{Na}_{3} \mathrm{P}$ and $\mathrm{c}$ ) the possible synthesis of $\mathrm{Li}_{5} \mathrm{P}$ and $\mathrm{Na}_{5} \mathrm{P}$ or other Li- or Na-rich phosphorous compounds under pressure. The results reveal an enhanced reactivity of both alkali metals with phosphorous at slightly elevated pressures, enabling the synthesis of $\mathrm{Li}_{3} \mathrm{P}$ and $\mathrm{Na}_{3} \mathrm{P}$ at RT starting from element precursors, bypassing the established complicated chemical synthesis methods. A pressure induced phase transition towards a $F m \overline{3} m$ (FCC) structure, that remains stable up to the highest pressure of this study, was observed for both compounds. For both compounds, the $F m \overline{3} m$ phase transforms back to the $P 6_{3} / m m c$ phase (stable at ambient conditions) upon pressure release, with negligible hysteresis. Finally, we unsuccessfully attempted to synthesize compounds with higher alkali metal content using high-temperature and -pressure conditions.

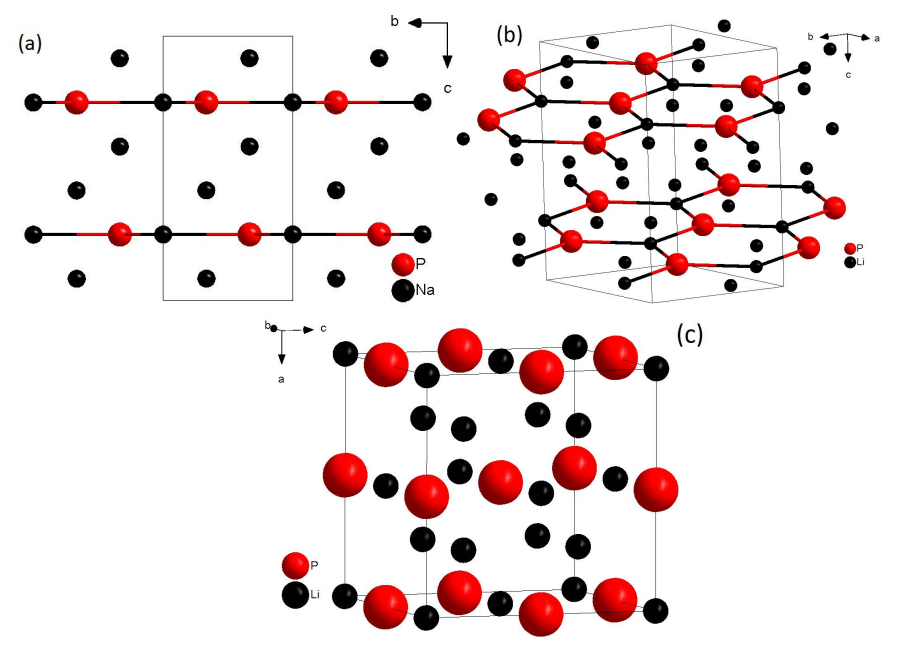

Fig. 1 a) and b) Schematic representations of the ambient conditions crystal structure of $(\mathrm{Na} / \mathrm{Li})_{3} \mathrm{P}$. c) Schematic representations of the high-pressure FCC crystal structures of $(\mathrm{Na} / \mathrm{Li})_{3} \mathrm{P}$.

\section{METHODS}

\subsection{Experimental Methods}

High purity, commercially available lithium (Sigma-Aldrich, $>99.9 \%$ purity), sodium (Sigma-Aldrich, $>99.95 \%$ purity) and amorphous red phosphorous (Sigma-Aldrich, $>99.9 \%$ purity) were used for the synthesis experiments in this study. Rhenium gaskets (preindented to 40-50 $\mu \mathrm{m}$ thick using 400-500 $\mu \mathrm{m}$ diamond culets) were used. Initial sample chamber diameters were nominally 100-200 $\mu \mathrm{m}$. Red phosphorous was ground to a fine powder for the angle-dispersive X-ray diffraction (XRD) measurements and was loaded into a diamond anvil cell (DAC), filling about 10-25\% of the DAC cavity volume. The remaining volume was filled with either Li or $\mathrm{Na}$ and small quantities of pressure sensors (gold and ruby) inside a glovebox at room temperature (RT). Pressure was determined through ruby luminescence ${ }^{19}$, gold equation of state
(EOS $)^{20}$ and the previously published EOSs of $\mathrm{Na}^{21}$ and $\mathrm{Li}^{22}$. All readings were in agreement within $1 \mathrm{GPa}$ at pressures $>10 \mathrm{GPa}$.

A Pilatus $1 \mathrm{M}$ CdTe detector was used at the undulator X-ray diffraction (XRD) Beamline 13-ID-D at GeoSoilEnviroCARS, sector13, APS, Chicago; a Pilatus 1M Si detector was used at the undulator X-ray diffraction (XRD) Beamline 16-IDB at High Pressure Collaborative Access Team (HPCAT), at the Advanced Photon Source (APS); and a MAR-345 image plate detector was used at the Advanced Light Source, Lawrence Berkeley National Laboratory Beamline 12.2.2 to collect pressure dependent X-ray diffraction data. The X-ray probing beam spot size was focused to approximately $2-4 \mu \mathrm{m}$ at GeoSoilEnviroCARS and HPCAT and to $10 \mu \mathrm{m}$ at beamline 12.2.2 using Kirkpatrick-Baez mirrors. Additional details on the XRD experimental setups are given in Prakapenka et al. ${ }^{23}$ and Kunz et al. ${ }^{24}$. XRD data were collected in situ at high temperature and on the quenched samples. Double-sided CW laser heating (LH) was performed using ytterbium (Yb:YAG) fiber laser $1064 \mathrm{~nm}$ wavelength) focused to a flat top around 10-30 $\mu \mathrm{m}$ in diameter (FWHM) spot ${ }^{23,24}$. Temperature was measured spectroradiometrically simultaneously with XRD measurements with a typical uncertainty of $150 \mathrm{~K}^{23,24}$. Integration of powder diffraction patterns to yield scattering intensity versus $2 \theta$ diagrams and initial analysis were performed using the DIOPTAS program ${ }^{25}$. Calculated XRD patterns were produced using the POWDER CELL program ${ }^{26}$, for the corresponding crystal structures according to the EOSs determined experimentally and theoretically in this study and assuming continuous Debye rings of uniform intensity. Indexing of XRD patterns was performed using the DICVOL program ${ }^{27}$ as implemented in the FullProf Suite.

\subsection{Computational methods}

The $a b$ initio random structure searching (AIRSS) technique 28,29 is used to search for candidate structures. AIRSS is a stochastic method which generates structures randomly with a given number of formula units. A minimum atom-atom separation is specified for the generated structures. These separations are chosen based on short AIRSS runs. Symmetry constraints are imposed on our generated structures. This strategy tends to speed up the searches because low-symmetry structures are unlikely to have low energies according to Pauling's principle ${ }^{30,31}$, although we allocate part of our searching time to check low symmetry structures, for completeness. The generated structures are relaxed using first-principles quantum mechanical density functional theory (DFT) methods as implemented in the CASTEP code. ${ }^{32}$ Computational details and all predicted structures could be found in https://doi.org/10.6084/m9.figshare.12866975. This procedure is carried out in parallel to ensure a high throughput of results. AIRSS has a proven track record of predicting structures in a diverse variety of systems that have subsequently been verified by experiment, such as in compressed silane, ${ }^{28}$ aluminium hydride, ${ }^{33}$ high-pressure hydrogen sulfide, ${ }^{34}$ and xenon oxides. ${ }^{35}$ We limit our searches to a maximum of eight formula units 32 atoms) per cell. A plane-wave basis set energy cutoff of $340 \mathrm{eV}$ was used for the structure searches, and a cutoff of $700 \mathrm{eV}$ was used for the final reported converged results. The Perdew-Burke-Ernzerhof general- 
ized gradient approximation (GGA-PBE) ${ }^{36}$ exchange correlation functional was used, as implemented in CASTEP code ${ }^{32}$. The Brillouin zone was sampled using a Monkhorst-Pack k-point grid $^{37}$ of spacing $2 \pi \times 0.07 \AA^{-1}$ for the structure searches, and a k-point mesh with $2 \pi \times 0.07 \AA^{-1}$ spacing for the final converged results.

\section{Results and Discussion}

\subsection{Synthesis of $\mathrm{Na}_{3} \mathrm{P}$ and $\mathrm{Li}_{3} \mathrm{P}$ under pressure}

Synthesis of $\mathrm{Na}_{3} \mathrm{P}$ and $\mathrm{Li}_{3} \mathrm{P}$ usually requires extended and complicated chemical procedures, including prolonged stepwise heating $>400{ }^{\circ} \mathrm{C}$ of elements or precursors (e.g. $\mathrm{LiP}+\mathrm{Li}$ ) in an autoclave ${ }^{38-40}$. At ambient conditions both $\mathrm{Na}_{3} \mathrm{P}$ and $\mathrm{Li}_{3} \mathrm{P}$ compounds crystallize in a hexagonal structure $\left(\mathrm{Na}_{3}\right.$ As-type, space group: $P 6_{3} / m m c$ (194)) with two formula units $(\mathrm{Z}=2)$ per unit cell ${ }^{39,41-43}$. The main structural characteristic of this structure is the presence of $\mathrm{Na}$ /Li-P graphite-like layers (in $\mathrm{AB}$ stacking) intercalated by two layers of $\mathrm{Na} / \mathrm{Li}$ atoms ${ }^{41,42}$, see Figures 1 (a),(b). P atoms are five-fold coordinated by $\mathrm{Na} / \mathrm{Li}$ atoms forming $\mathrm{P}(\mathrm{Na} / \mathrm{Li})_{5}$ trigonal bipyramids linked by corner sharing.

In this study, the Na-P and Li-P mixtures were sealed in DACs inside a glovebox (argon filled) at RT and the pressure was slightly increased to prevent any exposure of $\mathrm{Na}$ and $\mathrm{Li}$ to atmospheric air during the XRD experiments. More than 4 independent loadings were prepared for each mixture, with varying initial pressure in the range of 0.8-3 GPa. The XRD measurements were performed a few days (one to four) after initial pressure increase. In all independent loadings a direct reaction between Na-P and Li-P was observed towards the formation of $\mathrm{Na}_{3} \mathrm{P}$ and $\mathrm{Li}_{3} \mathrm{P}$, respectively. The $\mathrm{XRD}$ patterns of the synthesized compounds are in excellent agreement with the known crystal structures of $\mathrm{Na}_{3} \mathrm{P}$ and $\mathrm{Li}_{3} \mathrm{P}$ at ambient conditions, see Figure 2. Detailed XRD mapping of the samples inside the DAC revealed a complete reaction of $\mathrm{P}$, while an excess of $\mathrm{Na}$ and Li remained. Moreover, the size and the morphology of the synthesized $\mathrm{Na}_{3} \mathrm{P}$ and $\mathrm{Li}_{3} \mathrm{P}$ are practically identical with the starting piece of the powdered red-P. Given that the melting lines of both $\mathrm{Na}$ and Li are well above RT in the 0-3 GPa pressure range ${ }^{44-47}$, it is plausible to assume a solid-solid reaction between $\mathrm{Na} / \mathrm{Li}$ and $\mathrm{P}$.

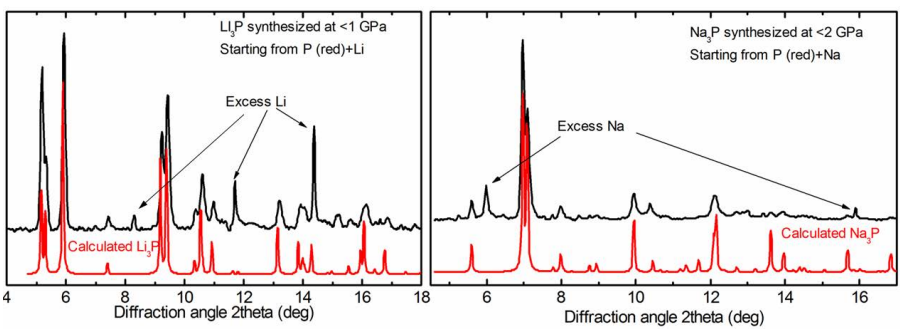

Fig. 2 Diffraction patterns of the synthesized $\mathrm{Li}_{3} \mathrm{P}$ (left) and $\mathrm{Na}_{3} \mathrm{P}$ (right) in comparison to the calculated diffraction patterns of the published crystal structures of $\mathrm{Li}_{3} \mathrm{P}$ and $\mathrm{Na}_{3} \mathrm{P}^{41,42}$. The $\mathrm{x}$-ray wavelengths are $\lambda=0.3344 \AA$ for $\mathrm{Li}_{3} \mathrm{P}$ and $\lambda=0.2952 \AA$ for $\mathrm{Na}_{3} \mathrm{P}$.

The critical pressures (lowest limit) for the synthesis of $\mathrm{Li}_{3} \mathrm{P}$ and $\mathrm{Na}_{3} \mathrm{P}$ was not examined in detail, due to the experimental conditions that precluded in-situ XRD measurements upon initial pressure increase inside the glovebox. However, in both mixtures suc- cessful synthesis was achieved for the lowest starting pressure for each mixture i.e. $0.8 \mathrm{GPa}$ for Li-P and $1 \mathrm{GPa}$ for Na-P. Upon full pressure release both the $\mathrm{Li}_{3} \mathrm{P}$ and $\mathrm{Na}_{3} \mathrm{P}$ compounds remained stable.

\subsection{High pressure structural study of $\mathrm{Li}_{3} \mathrm{P}$}

After successful synthesis of $\mathrm{Li}_{3} \mathrm{P}$ the pressure was increased up to $20 \mathrm{GPa}$. Figure 3 shows integrated diffraction patterns of $\mathrm{Li}_{3} \mathrm{P}$ at selected pressures. The evolution of the $\mathrm{Li}_{3} \mathrm{P}$ XRD data shows discontinuous changes $>6 \mathrm{GPa}$, revealing the occurrence of a phase transition. The previously reported ${ }^{22}$ b.c.c. $\rightarrow$ f.c.c. transition of Li was also observed near 7.5 GPa. The observed Bragg peaks of the high-pressure phase of $\mathrm{Li}_{3} \mathrm{P}$ can be very well indexed with the $F m \overline{3} m$ (FCC) structure predicted by Zhao et al. ${ }^{15}$ (Li ${ }_{3}$ Sb-type, S.G.: $F m \overline{3} m$ (225), $Z=4$ ) with $a=5.565 \AA$ at $13 \mathrm{GPa}$, see Figure 4(a). The phase transition is likely induced by a collapse of the $P 6_{3} / m m c$ phase c-axis, combined with the lithium atoms aligning into a single plane and a slight shift of the phosphorous atoms to a symmetric spacing, see Figure 1(c).

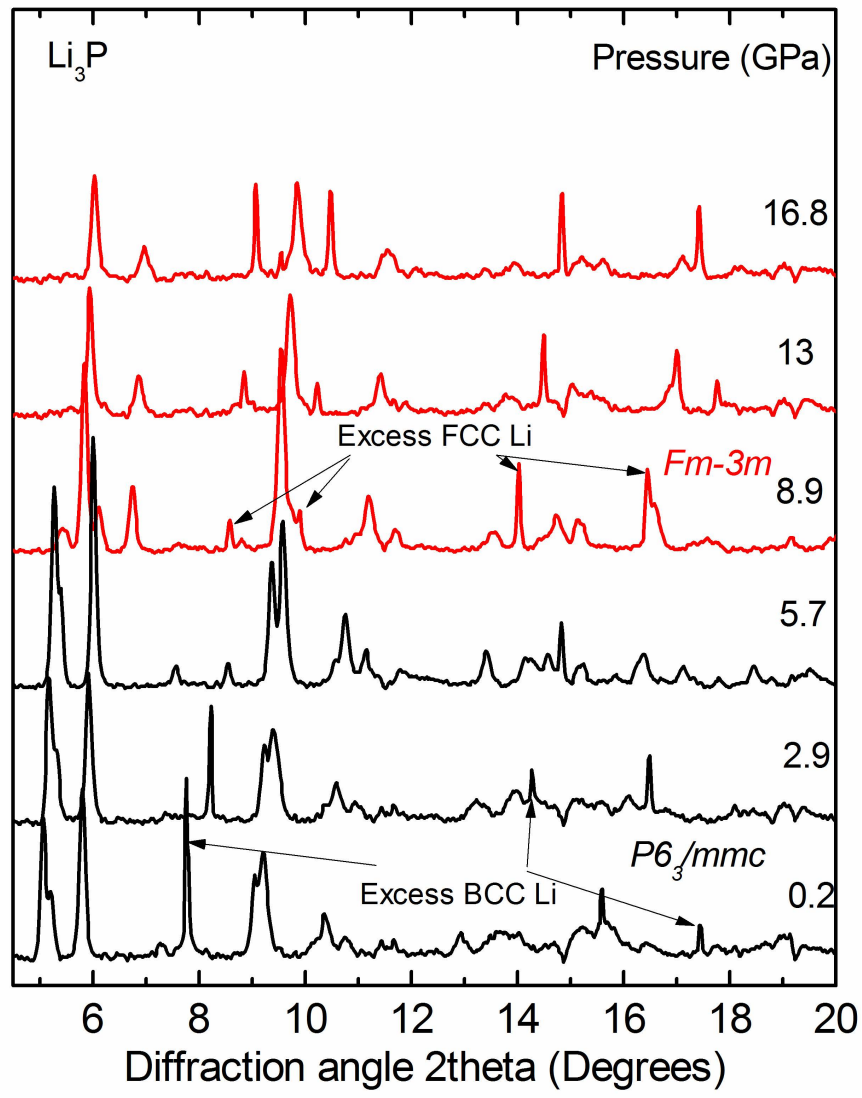

Fig. 3 Diffraction patterns of $\mathrm{Li}_{3} \mathrm{P}$ and excess of $\mathrm{Li}$ at selected pressures measured on pressure increase. The XRD patterns of the $P 6_{3} / \mathrm{mmc}$ and $F m \overline{3} m$ phases are shown by black and red, respectively. The x-ray wavelengths is $\lambda=0.3344 \AA$.

From the XRD data of $\mathrm{Li}_{3} \mathrm{P}$, we have obtained lattice parameters and the cell volume per formula unit $\left(\mathrm{V}_{\text {p.f.u. }}\right)$ of the two $\mathrm{Li}_{3} \mathrm{P}$ structures as a function of pressure. The results are presented in Figure 5. The plot of relative $\mathrm{V}_{\text {p.f.u. }}$. versus pressure show a volume reduction of $9 \%$ for the $P 6_{3} / m m c$ to FCC transi- 

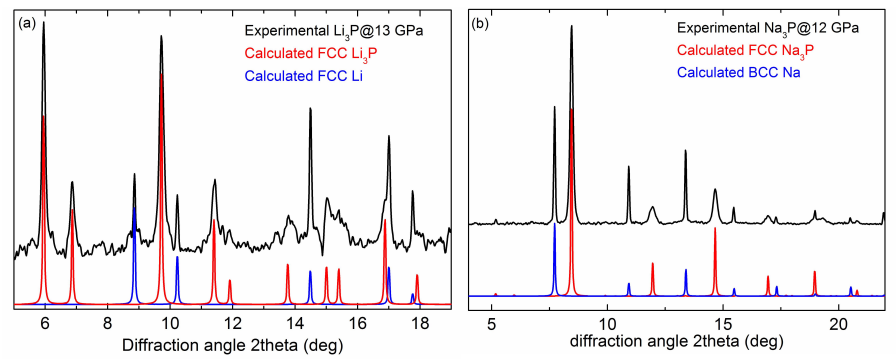

Fig. 4 a) Comparison between the experimental pattern of the $\mathrm{Li}_{3} \mathrm{P}+\mathrm{Li}$ mixture at $13 \mathrm{GPa}$ and the calculated pattern of $F m \overline{3} m \mathrm{Li}_{3} \mathrm{P}$ and $\mathrm{FCC} \mathrm{Li}$ and b) Comparison between the experimental pattern of the $\mathrm{Na}_{3} \mathrm{P}+\mathrm{Na}$ mixture at $12 \mathrm{GPa}$ and the calculated pattern of $F m \overline{3} m \mathrm{Na}_{3} \mathrm{P}$ and BCC $\mathrm{Na}$. The x-ray wavelength is $\lambda=0.3344 \AA$.

tion at $6 \mathrm{GPa}$. Usually, such large volume decrease is indicative of major atomic rearrangements which in this case involve the rearrangement of the two Li layers into one. We have fitted a thirdorder Birch-Murnaghan equation of state ${ }^{48}$ to the experimental pressure-volume data and determined the bulk modulus $B$ and its first derivative $B^{\prime}$ at zero pressure for the $P 6_{3} / m m c$ and at the experimental onset pressure for the FCC phase. The elastic parameters obtained in this way are given in Table 1.

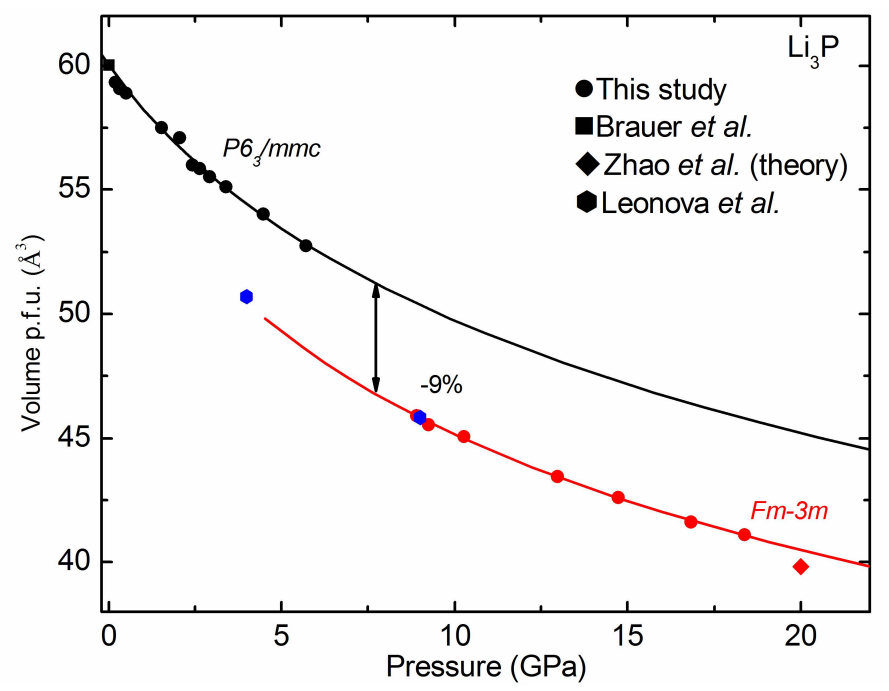

Fig. 5 Volume-pressure data for the $P 63 / m m c$ and $F m \overline{3} m$ phases of $\mathrm{Li}_{3} \mathrm{P}$ as determined in this study together with the results from Refs. 43 (ambient conditions), 18 ( $\mathrm{P}=4 \mathrm{GPa}, \mathrm{T}=700^{\circ} \mathrm{C}$ and $\left.\mathrm{P}=9 \mathrm{GPa}, \mathrm{T}=\mathrm{RT}\right)$ and 15 (calculated $F m \overline{3} m$ ). The solid lines are unweighted third-order Birch-Murnaghan EOS ${ }^{48}$ fits to the experimental data points.

\subsection{Attempts to synthesize $\mathrm{Li}_{5} \mathrm{P}$ under pressure}

Two independent approaches were followed aiming to synthesize $\mathrm{Li}_{5} \mathrm{P}$ (or Li-rich compounds in general): a) prolonged heating at temperatures of $\sim 200{ }^{\circ} \mathrm{C}$ and $\mathrm{b}$ ) short (few seconds) heating at temperatures $>1000 \mathrm{~K}$. In both cases, the pressure of the $\mathrm{Li}_{3} \mathrm{P}+\mathrm{Li}$ mixture was increased at various pressures above $10 \mathrm{GPa}$, i.e. the predicted pressure by Zhao et al. ${ }^{15}$ above which $\mathrm{Li}_{5} \mathrm{P}$ becomes stable, and up to max $20 \mathrm{GPa}$ to avoid failure of diamond anvils ${ }^{22}$. In the case of prolonged heating, the DACs were placed inside a
Table 1 Experimental and calculated structural parameters of $P 6_{3} / \mathrm{mmc}$ and $F m \overline{3} m$ phases of $\mathrm{Li}_{3} \mathrm{P}$ at selected pressures: space group (SG), number of formula units in the unit cell $Z$, lattice parameters, volume per formula unit, bulk modulus B and its pressure derivative B'.

\begin{tabular}{cccccccc}
$\mathrm{P}(\mathrm{GPa})$ & $\mathrm{SG}$ & $\mathrm{Z}$ & $a(\AA)$ & $c(\AA)$ & $V_{p . f . u .}\left(\AA^{3}\right)$ & $\mathrm{B}(\mathrm{GPa})$ & $\mathrm{B}^{\prime}$ \\
\hline 0 Brauer et al. ${ }^{43}$ & $P 6_{3} / m m c$ & 2 & 4.2730 & 7.5940 & 60.04 & & \\
1.54 & $P 6_{3} / m m c$ & 2 & $4.210(1)$ & $7.492(3)$ & $57.48(4)$ & $30(3)$ & $6(2)$ \\
\hline 13 & $F m \overline{3} m$ & 4 & $5.565(4)$ & & $43.08(7)$ & $65(3)$ & $5(1)$ \\
20 Zhao et al. ${ }^{15}$ & $F m \overline{3} m$ & 4 & 5.4216 & & 39.84 & & \\
\hline
\end{tabular}

furnace at temperatures of $\sim 200^{\circ} \mathrm{C}$ for more than $48 \mathrm{~h}$, while XRD measurements aiming to trace any reaction were performed every 12h. Laser heating was used for the high-temperature attempts and we experienced several failures of diamond anvils especially at pressures close to $20 \mathrm{GPa}$. Nevertheless, we were able to perform few synthesis attempts at pressures between 15 to $20 \mathrm{GPa}$ and temperatures between $1000-2000 \mathrm{~K}$. No sign of the formation of a new compound was observed in either of the approaches and in all combinations of pressure and temperature while, $\mathrm{Li}_{3} \mathrm{P}$ remained stable even at high temperatures.

\subsection{High pressure structural study of $\mathrm{Na}_{3} \mathrm{P}$}

After successful synthesis of $\mathrm{Na}_{3} \mathrm{P}$ the pressure was increased up to $20 \mathrm{GPa}$. Figure 6 shows integrated diffraction patterns of $\mathrm{Na}_{3} \mathrm{P}$ at selected pressures. The evolution of the $\mathrm{Na}_{3} \mathrm{P}$ XRD data shows discontinuous changes $>5 \mathrm{GPa}$, revealing the occurrence of a phase transition between 4 to $6 \mathrm{GPa}$, depending on the time scale of pressure increase. A preliminary indexing of the XRD pattern at $12 \mathrm{GPa}$ shows that it has a I-type cubic cell with $\mathrm{Z}=1$ (f. u. per unit cell). However, this solution is not conclusive without the knowledge of atomic positions in the unit cell.

To address the structure, we have carried out first principles structural searches using AIRSS. The AIRSS results revealed that a $F m \overline{3} m$ (FCC) structure, of the same structural type ( $\mathrm{Li}_{3} \mathrm{Sb}$-type, S.G.:F $m \overline{3} m$ (225), $\mathrm{Z}=4$ ) as the one predicted by Zhao et al. ${ }^{15}$ in the case of $\mathrm{Li}_{3} \mathrm{P}$, is the most stable structure for $\mathrm{Na}_{3} \mathrm{P}$ above $5 \mathrm{GPa}$, see Fig. 7. The observed Bragg peaks of the high-pressure phase of $\mathrm{Na}_{3} \mathrm{P}$ can be very well indexed with the $F m \overline{3} m$ (FCC) structure with $\mathrm{a}=6.428 \AA$ at $12 \mathrm{GPa}$, see Figure 4(b).

From the XRD data of $\mathrm{Na}_{3} \mathrm{P}$, we have obtained the lattice parameters and the cell volume per formula unit $\left(\mathrm{V}_{\text {p.f.u. }}\right)$ for both of the two $\mathrm{Na}_{3} \mathrm{P}$ structures as functions of pressure. The results are presented in Figure 8. The plot of relative $\mathrm{V}_{p . f . u}$. versus pressure shows a volume reduction of $9.5 \%$ for the $P 6_{3} / m m c$ to f.c.c. transition at $4.4 \mathrm{GPa}$. We have fitted a third-order Birch-Murnaghan equation of state ${ }^{48}$ to the experimental pressure-volume data and determined the bulk modulus $B$ and its first derivative $B^{\prime}$ at zero pressure for the $P 6_{3} / m m c$ and at the experimental onset pressure for the FCC phase. The elastic parameters obtained in this way are given in Table 2 .

\subsection{Attempts to synthesize $\mathrm{Na}_{5} \mathrm{P}$ under pressure}

The same two main approaches followed in the case of the $\mathrm{Li}_{3} \mathrm{P}+\mathrm{Li}$ mixture were also used in the case of the $\mathrm{Na}_{3} \mathrm{P}+\mathrm{Na}$ mixture with the exception that the pressure was increased up to $30 \mathrm{GPa}$. Sim- 


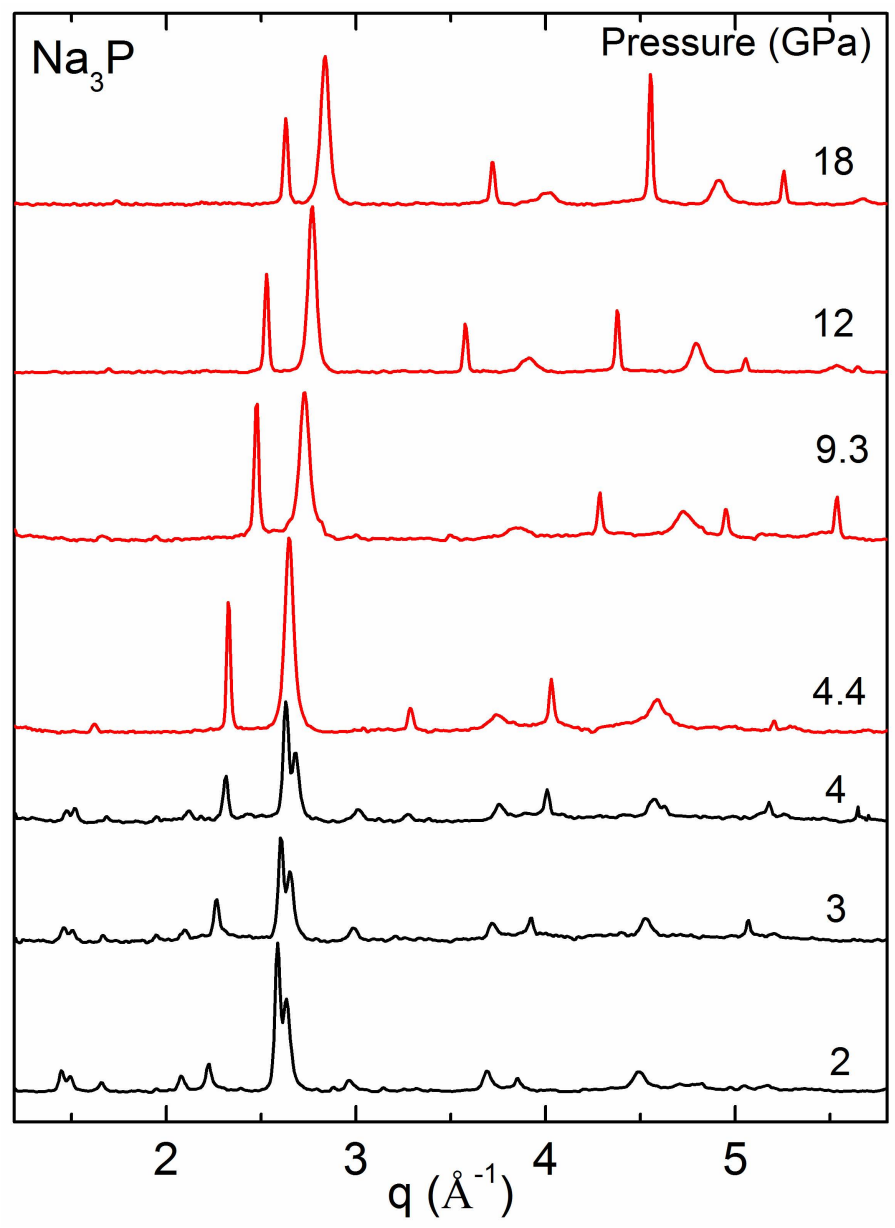

Fig. 6 Diffraction patterns of $\mathrm{Na}_{3} \mathrm{P}$ and excess of $\mathrm{Na}$ at selected pressures measured on pressure increase. The XRD patterns of the $P 6_{3} / m m c$ and $F m \overline{3} m$ phases are shown by black and red, respectively.

ilarly with $\mathrm{Li}_{3} \mathrm{P}+\mathrm{Li}$, no sign of the formation of a new compound was observed in either approach and in all combinations of pressure and temperature while, $\mathrm{Na}_{3} \mathrm{P}$ remained stable even at high temperatures.

\section{Conclusion}

The chemical reactivity of the Na-P and Li-P systems and the structural behavior of the relevant compounds were studied under high pressure conditions using synchrotron $\mathrm{x}$-ray diffraction in a diamond anvil cell up to $20 \mathrm{GPa}$ combined with $a b$ initio random structure searching technique. An enhanced reactivity of both alkali metals with phosphorous was observed at slightly elevated pressures facilitating the synthesis of $\mathrm{Li}_{3} \mathrm{P}$ and $\mathrm{Na}_{3} \mathrm{P}$ at RT bypassing established chemical synthesis methods. It is plausible to assume that the slight increase of pressure substantially decreases the relevant energy barriers, facilitating the relevant chemical reactions at RT. A pressure-induced phase transition towards a $F m \overline{3} m$ (FCC) structure that remains stable up to $20 \mathrm{GPa}$ was observed, supported by the random structure searching techniques used in this and previous studies. In contrast to recent theoretical predictions, the attempts to synthesize compounds with higher alkali metal content were not successful. Further theoretical studies are

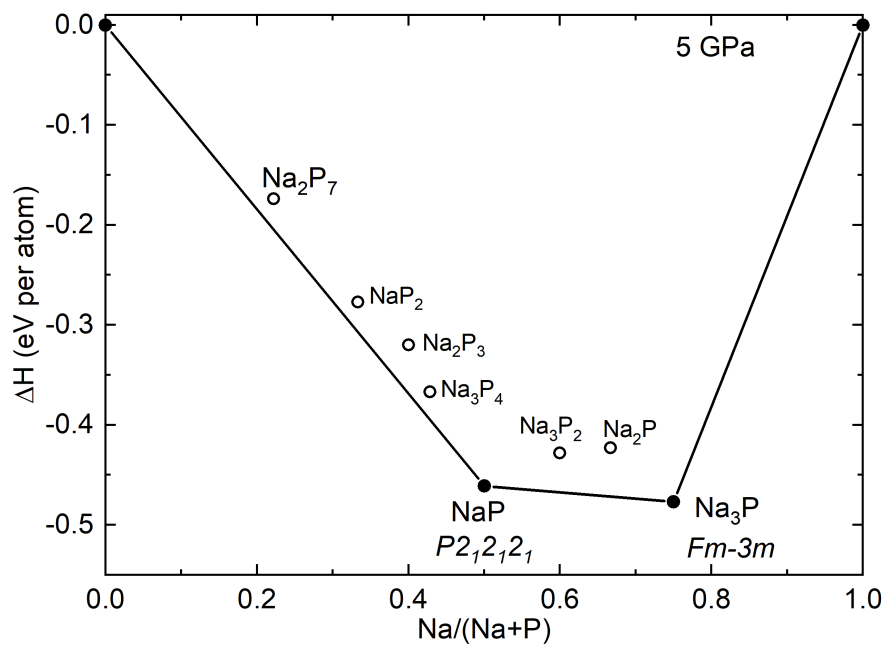

Fig. 7 Convex hull diagram of the Na-P system at $5 \mathrm{GPa}$. Solid circles represent stable phases and open circles metastable phases. The crystal structures of of the stable phases are noted.

Table 2 Experimental and calculated structural parameters of $P 6_{3} / \mathrm{mmc}$ and $F m \overline{3} m$ phases of $\mathrm{Na}_{3} \mathrm{P}$ at selected pressures: space group (SG), number of formula units in the unit cell $Z$, lattice parameters, volume per formula unit, bulk modulus B and its pressure derivative B'.

\begin{tabular}{|c|c|c|c|c|c|c|c|}
\hline $\mathrm{P}(\mathrm{GPa})$ & SG & Z & $a(\AA)$ & $c(\AA)$ & $V_{\text {p.f.u. }}\left(\AA^{3}\right)$ & $\mathrm{B}(\mathrm{GPa})$ & $\mathrm{B}^{\prime}$ \\
\hline 0 Brauer et al. ${ }^{43}$ & $P 6_{3} / m m c$ & 2 & 4.98 & 8.797 & 94.47 & & \\
\hline 2 & $P 6_{3} / m m c$ & 2 & $4.865(1)$ & $8.693(2)$ & $89.09(4)$ & $26.5(6)$ & 4 (fixed) \\
\hline 5.5 & $F m \overline{3} m$ & 4 & $6.639(4)$ & & $73.15(6)$ & $47.8(20)$ & $3.3(4)$ \\
\hline 5 (theory this study) & $F m \overline{3} m$ & 4 & 6.675 & & 74.352 & & \\
\hline
\end{tabular}

needed to completely elucidate the high-pressure chemical reactivity of the Na-P and Li-P systems. Moreover, it is possible that there is a large energy barrier for the $(\mathrm{Li} / \mathrm{Na})_{3} \mathrm{P}+2(\mathrm{Li} / \mathrm{Na}) \rightarrow(\mathrm{Li} / \mathrm{Na})_{5} \mathrm{P}$ reaction, requiring higher temperatures, or other approaches, to overcome the barrier. However, in the case of Li this will be experimentally challenging due to diamond anvils failure.

\section{Conflicts of interest}

A relevant US patent application (No.16/908492) on the experimental synthesis of the $\mathrm{Li}_{3} \mathrm{P}$ and $\mathrm{Na}_{3} \mathrm{P}$ compounds was submitted.

\section{Acknowledgments}

Part of this work was performed under the auspices of the U. S. Department of Energy by Lawrence Livermore National Security, LLC under Contract DE-AC52-07NA27344. We gratefully acknowledge the LLNL LDRD program for funding support of this project under 18-LW-036. Part of this work was performed at GeoSoilEnviroCARS (The University of Chicago, Sector 13), Advanced Photon Source (APS), Argonne National Laboratory. GeoSoilEnviroCARS is supported by the National Science Foundation - Earth Sciences (EAR-1634415) and Department of Energy-GeoSciences (DE-FG02-94ER14466). This research used resources of the Advanced Photon Source, a U.S. Department of Energy (DOE) Office of Science User Facility operated for the DOE Office of Science by Argonne National Laboratory under Contract No. DE-AC0206CH11357. The ALS is supported by the Director, Office of Sci- 


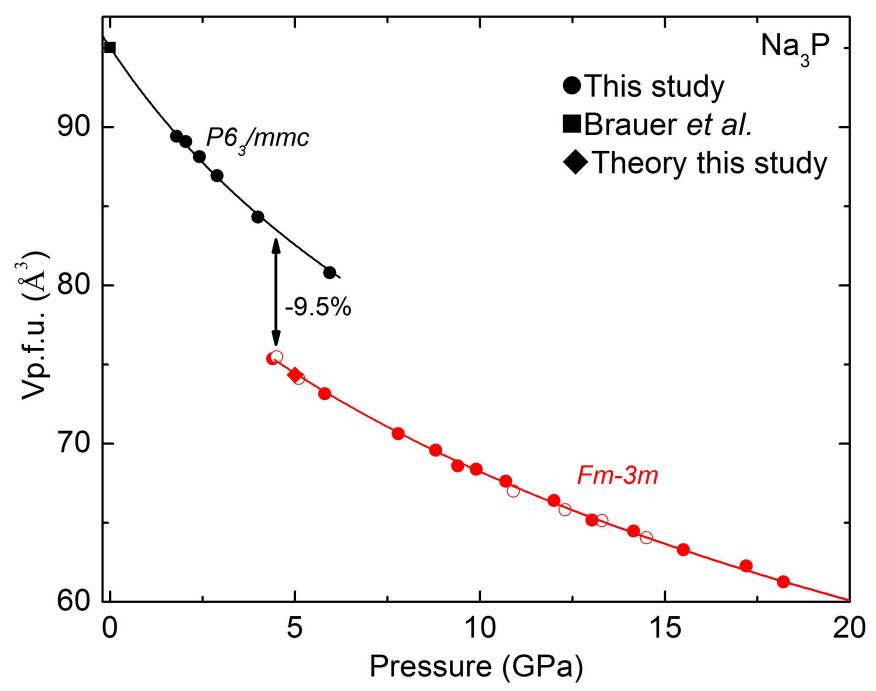

Fig. 8 Volume-pressure data for the $P 6_{3} / m m c$ and $F m \overline{3} m$ phases of $\mathrm{Na}_{3} \mathrm{P}$ as determined experimentally and theoretically in this study together with the results from Refs. 43 (ambient conditions). The solid lines are unweighted third-order Birch-Murnaghan EOS fits to the experimental data points ${ }^{48}$. Solid and open symbols correspond to the measurements on pressure increase and decrease, respectively.

ence, BES of DOE under Contract No. DE-AC02-05CH11231, DEAC02-06CH11357. Part of this this work was performed at HPCAT (Sector 16), Advanced Photon Source (APS), Argonne National Laboratory. HPCAT operations are supported by DOE-NNSAâÁŹs Office of Experimental Sciences. CJP is supported by the Royal Society through a Royal Society Wolfson Research Merit award, and UKCP consortium grant EP/P022596/1 from the EPSRC.

\section{References}

1 G. Q. X. Dong, A. R. Oganov, Q. Z. X-F. Zhou and H.-T. Wang, ArXiv e-prints, 2015, arXiv:1503.00230.

2 L. Zhu, H. Liu, C. J. Pickard, G. Zou and Y. Ma, Nat. Chem., 2014, 6, 644.

3 E. Stavrou, Y. Yao, A. F. Goncharov, S. S. Lobanov, J. M. Zaug, H. Liu, E. Greenberg and V. B. Prakapenka, Phys. Rev. Lett., 2018, 120, 096001.

4 X. Dong, A. R. Oganov, A. F. Goncharov, E. Stavrou, S. Lobanov, G. Saleh, G.-R. Qian, Q. Zhu, C. Gatti, V. L. Deringer, R. Dronskowski, X.-F. Zhou, V. B. Prakapenka, Z. KonÃtpkovÃą, I. A. Popov, A. I. Boldyrev and H.-T. Wang, Nat. Chem., 2017, 9, 440.

5 W. Zhang, A. R. Oganov, A. F. Goncharov, Q. Zhu, S. E. Boulfelfel, A. O. Lyakhov, E. Stavrou, M. Somayazulu, V. B. Prakapenka and Z. Konôpková, Science, 2013, 342, 15021505.

6 D. Laniel, G. Weck, G. Gaiffe, G. Garbarino and P. Loubeyre, J. Phys. Chem. Lett., 2018, 9, 1600-1604.

7 A. P. Drozdov, M. I. Eremets, I. A. Troyan, V. Ksenofontov and S. I. Shylin, Nature, 2015, 525, 73.
8 M. Somayazulu, M. Ahart, A. K. Mishra, Z. M. Geballe, M. Baldini, Y. Meng, V. V. Struzhkin and R. J. Hemley, Phys. Rev. Lett., 2019, 122, 027001.

9 A. R. Oganov, C. J. Pickard, Q. Zhu and R. J. Needs, Nature Reviews Materials, 2019, 4, 331-348.

10 L. E. Marbella, M. L. Evans, M. F. Groh, J. Nelson, K. J. Griffith, A. J. Morris and C. P. Grey, J. Am. Chem. Soc., 2018, 140, 79948004.

11 V. Palomares, P. Serras, I. Villaluenga, K. B. Hueso, J. Carretero-GonzÃąlez and T. Rojo, Energy Environ. Sci., 2012, 5, 5884-5901.

12 S. Y. Hong, Y. Kim, Y. Park, A. Choi, N.-S. Choi and K. T. Lee, Energy Environ. Sci., 2013, 6, 2067-2081.

13 M. Mayo, K. J. Griffith, C. J. Pickard and A. J. Morris, Chem. Mater., 2016, 28, 2011-2021.

14 F. Peng, M. Miao, H. Wang, Q. Li and Y. Ma, J. Am. Chem. Soc., 2012, 134, 18599-18605.

15 Z. Zhao, L. Liu, T. Yu, G. Yang and A. Bergara, J. Phys. Chem. C, 2017, 121, 21199-21205.

16 L. Hao, X. Li, Y. Zhang, K. Luo, Y. Gao, F. Ling, Y. Wu, Z. Zhao and D. Yu, Comp. Mat. Science, 2019, 158, 255 - 259.

17 Z. Zhao, S. Zhang, T. Yu, H. Xu, A. Bergara and G. Yang, Phys. Rev. Lett., 2019, 122, 097002.

18 M. E. Leonova, I. K. Bdikin, S. A. Kulinich, O. K. Gulish, L. G. Sevast'yanova and K. P. Burdina, Inorg. Mater., 2003, 39, 266270.

19 K. Syassen, High Pres. Res., 2008, 28, 75.

20 M. Matsui, International Conference On High Pressure Science and Technology, Joint AIRAPT-22 and HPCJ-50, 2010, 215, 012197.

21 M. Hanfland, I. Loa and K. Syassen, Phys. Rev. B, 2002, 65, 184109.

22 M. Hanfland, I. Loa, K. Syassen, U. Schwarz and K. Takemura, Solid State Commun., 1999, 112, 123 - 127.

23 V. B. Prakapenka, A. Kubo, A. Kuznetsov, A. Laskin, O. Shkurikhin, P. Dera, M. L. Rivers and S. R. Sutton, High Pres. Res., 2008, 28, 225-235.

24 M. Kunz, A. MacDowell, W. Caldwell, D. Cambie, R. Celestre, E. Domning, R. Duarte, A. Gleason, J. Glossinger, N. Kelez, D. Plate, T. Yu, J. Zaug, H. Padmore, R. Jeanloz, A. Alivisatos and S. Clark, J. Synchrotron Radiat., 2005, 12, 650.

25 C. Prescher and V. B. Prakapenka, High Pres. Res., 2015, 35, 223-230.

26 W. Kraus and G. Nolze, J. Appl. Crystallogr., 1996, 29, 301303. 
27 A. Boultif and D. Louër, J. Appl. Crystallogr., 2004, 37, 724731.

28 C. J. Pickard and R. Needs, Physical Review Letters, 2006, 97, 045504.

29 C. J. Pickard and R. J. Needs, J. Phys.: Condens. Matter, 2011, 23, 053201.

30 R. J. Needs and C. J. Pickard, APL Mater., 2016, 4, 053210.

31 L. Pauling, J. Am. Chem. Soc., 1929, 51, 1010-1026.

32 S. J. Clark, M. D. Segall, C. J. Pickard, P. J. Hasnip, M. I. Probert, K. Refson and M. C. Payne, Zeitschrift für Kristallographie-Crystalline Materials, 2005, 220, 567-570.

33 C. J. Pickard and R. Needs, Physical Review B, 2007, 76, 144114.

34 I. Errea, M. Calandra, C. J. Pickard, J. Nelson, R. J. Needs, Y. Li, H. Liu, Y. Zhang, Y. Ma and F. Mauri, Physical Review Letters, 2015, 114, 157004.

35 A. Dewaele, N. Worth, C. J. Pickard, R. J. Needs, S. Pascarelli, O. Mathon, M. Mezouar and T. Irifune, Nat. Chem., 2016, 8, 784-790.

36 J. P. Perdew, K. Burke and M. Ernzerhof, Phys. Rev. Lett., 1996, 77, 3865.
37 H. J. Monkhorst and J. D. Pack, Phys. Rev. B, 1976, 13, 51885192.

38 R. F. Jarvis, R. M. Jacubinas and R. B. Kaner, Inorg. Chem., 2000, 39, 3243-3246.

39 G. Nazri, Solid State Ionics, 1989, 34, 97 - 102.

40 Y. Xie, H. Su, B. Li and Y. Qian, Mater. Res. Bull., 2000, 35, 675 -680 .

41 Y. Dong and F. J. DiSalvo, Acta Cryst. E, 2005, 61, i223-i224.

42 Y. Dong and F. J. DiSalvo, Acta Cryst. E, 2007, 63, i97-i98.

43 G. Brauer and E. Zintl, Z. Phys. Chem., 1937, 37B, 323.

44 C.-S. Zha and R. Boehler, Phys. Rev. B, 1985, 31, 3199-3201.

45 H. D. Luedemann and G. C. Kennedy, J. Geophys. Res., 1968, 73, 2795-2805.

46 E. Gregoryanz, O. Degtyareva, M. Somayazulu, R. J. Hemley and H.-k. Mao, Phys. Rev. Lett., 2005, 94, 185502.

47 A. M. J. Schaeffer, W. B. Talmadge, S. R. Temple and S. Deemyad, Phys. Rev. Lett., 2012, 109, 185702.

48 F. Birch, J. Geophys. Res., 1978, 83, 1257-1268. 